\title{
Revisiting the Extended X-ray Absorption Fine Structure Fitting Procedure through a Machine Learning-Based Approach
}

\author{
A. Martini,* A. L. Bugaev,* S. A. Guda, A. A. Guda, E. Priola, E. Borfecchia, S. Smolders, K. Janssens, \\ D. De Vos, and A. V. Soldatov
}

\begin{abstract}
A novel approach for the analysis of extended X-ray absorption fine structure (EXAFS) spectra is developed exploiting an inverse machine learning-based algorithm. Through this approach, it is possible to explore and account for, in a precise way, the nonlinear geometry dependence of the photoelectron backscattering phases and amplitudes of single and multiple scattering paths. In addition, the determined parameters are directly related to the $3 \mathrm{D}$ atomic structure, without the need to use complex parametrization as in the classical fitting approach. The applicability of the approach, its potential and the advantages over the classical fit were demonstrated by fitting the EXAFS data of two molecular systems, namely, the $\mathrm{KAu}(\mathrm{CN})_{2}$ and the $\left[\mathrm{RuCl}_{2}(\mathrm{CO})_{3}\right]_{2}$ complexes.
\end{abstract}

\section{INTRODUCTION}

Extended X-ray absorption fine structure (EXAFS) spectroscopy is an efficient element selective technique employed for the extraction of local atomic structural parameters, such as interatomic distances, coordination numbers, and DebyeWaller (DW) factors. ${ }^{1,2}$ In the experimental procedure, X-ray photons are used to excite a core-level electron whose final state is modified because of its backscattering from neighboring atoms, resulting in the oscillatory behavior of the X-ray absorption coefficient, $\mu(E)$, as a function of photon energy. The oscillatory part $\chi(k)$, extracted from the $\mu(E)$ signal, can be described as a sum of an infinite number of scattering events $\chi_{i}(k):^{1,3}$

$$
\chi(k)=\sum_{i=1}^{\infty} \chi_{i}(k)
$$

with $\chi_{i}(k)$ defined as:

$$
\chi_{i}(k)=S_{0_{i}}^{2} \frac{N_{i} F_{i}(k)}{k R_{i}^{2}} \sin \left[2 k R_{i}+\phi_{i}(k)\right] e^{-2 \sigma_{i}^{2} k^{2}} \mathrm{e}^{-2 R_{i} / \lambda_{i}(k)}
$$

where $k$ denotes the excited photoelectron wavevector, $2 R_{i}$ is the total scattering path length, $N_{i}$ is the number of scattering paths equivalent to the selected one (i.e., the path degeneracy), and $\sigma_{i}^{2}$ is the DW factor associated with the $i^{\text {th }}$ scattering event. The terms $F_{i}$ and $\phi_{i}$ are the path backscattering amplitude factor and phase shift. $S_{0 i}^{2}$ is a many-body reduction factor accounting for the amplitude damping because of multi-

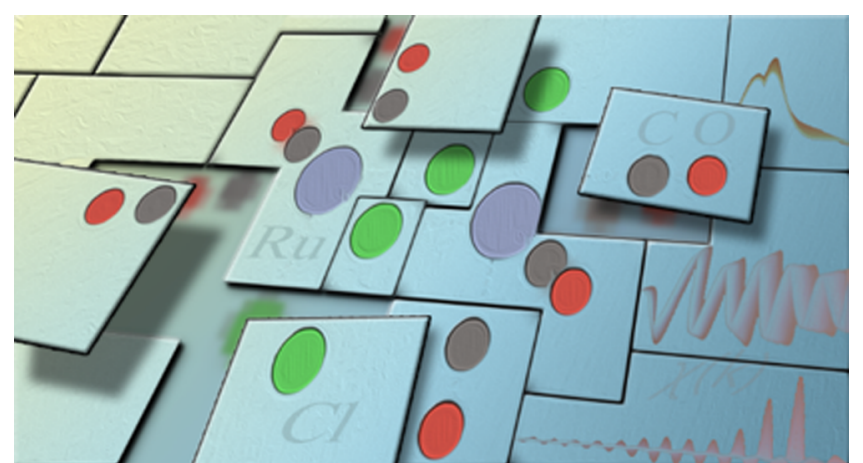

electron effects (intrinsic losses), while $\lambda_{i}(k)$ is the mean free path of the $i^{\text {th }}$ photoelectron.

Equation 1 involves different contributions stemming from single scattering (SS) paths, when the photoelectron is backscattered by one neighboring atom, and multiple scattering (MS) paths, when several atoms are involved in the scattering process. The latter ones contain information not only about the bond distances but also about the bond angles. However, because of the typically large path length, their contribution becomes significant only at high $k$-values because of the characteristic behavior of $\lambda_{i}(k) .{ }^{4}$ For practical implementation, the infinite series in eq 1 is always limited by the half path length $R_{i}$ and by the significance of the $\chi_{i}(k)$ contribution on the resulting EXAFS signal. ${ }^{5}$ In the typical implementation provided by the Artemis code (IFEFFIT), ${ }^{6}$ an initial guess structure is used for the $a b$ initio calculation of the scattering phases and amplitudes using the FEFF program, ${ }^{7}$ which are subsequently used to fit the experimental oscillatory signal given by eq 1 refining $S_{0}^{2}$ and $R_{i}$ and $N_{\mathrm{i}}$ and $\sigma^{2} \mathrm{i}$ for each selected path of eq 2 .

Although being well developed and successfully applied over decades, the standard approach in the EXAFS analysis exhibits 
some drawbacks. First, it is important to consider that the scattering amplitudes, phases, and electron mean free paths depend on the actual atomic structure and become unreliable for big variations of the bond distances with respect to the initial guess structure. In addition, it is worth mentioning that for the MS paths, which are in many cases neglected in the analysis, these functions show a strong nonlinear angular dependence especially in the case of linear atomic chains. The first attempt to solve this problem was provided by Frenkel et al. ${ }^{9-12}$ In these studies, the authors were able to estimate, from the analysis of certain double and triple forward MS paths, the average buckling angles of some mixed ionic salts . In particular, they demonstrated that it is possible to describe the buckling dependence for angles $\lesssim 20^{\circ}$ by the expansion of the effective backscattering amplitudes factors of these MS paths around the average buckling angles obtained by diffraction techniques. Further improvements to this problem were also provided in the $N$-body expansion of the GNXAS code ${ }^{13,14}$ and by alternative methodologies represented by the classical (force-field based) molecular dynamics $^{15-17}$ or by the reverse Monte Carlo approaches. ${ }^{18,19}$

The second problem is related to the choice of variables, appearing in eq 2 , which can be properly refined through the fitting procedure, because the maximum number of fitting parameters is limited according to the Nyquist criterion to $N_{i \mathrm{dp}} \approx 2 \frac{\Delta k \Delta R}{\pi}$, where $\Delta R$ and $\Delta k$ are the fitting range in the direct $(R)$ space and the $k$-interval used for the Fourier transform, respectively. ${ }^{20,21}$ Furthermore, as the number of parameters involved in the fit increases, the correlation among all the couples of the fitting variables becomes higher. These two facts frequently lead to the necessity to express some fitting variables, especially those linked to MS paths in eq 2, as a function of parameters already involved in the EXAFS fit within a simpler SS model. ${ }^{22}$ Apart from technical complications and the absence of a common modus operandi in the field, the conversion of the fitting parameters into the exact $3 \mathrm{D}$ representation of the atomic structure is not straightforward.

In the pioneering studies of Timoshenko et al., ${ }^{23,24}$ the possibility to exploit a machine learning $(\mathrm{ML})$ approach for the quantitative analysis of EXAFS spectra was demonstrated. In particular, the authors have constructed certain artificial neuralnetworks that allowed predicting, starting from certain experimental spectra, the two-body radial distribution functions (also partial) related to different atoms composing metal bulk materials and mono/bimetallic nanoparticles.

Herein, we propose an alternative EXAFS fitting procedure exploiting an indirect ML-based approach. The term indirect ${ }^{25}$ is used to indicate that a certain ML algorithm is employed to predict, from a certain set of user-defined structural parameters, those path signals assumed to influence more the related experimental EXAFS spectrum. More specifically, our method extends the multidimensional interpolation approach, proposed originally by Smolentsev et al. in the FitIt code ${ }^{26}$ and recently improved by our group in the new PyFitIt code, ${ }^{27}$ from the XANES to the EXAFS case. A theoretical training set is used to construct a series of implicit functions approximating each scattering path $\chi_{i}(k)$ under the continuous variation of some predefined parameters. Equation 1 is then generalized to account for any kind of deformations: from the bonds stretching till to the variation of multiple angles of the selected molecular structure, including significant deviations from the initial molecular geometry. Moreover, the effective path lengths associated with each scattering process are obtained directly as a function of the selected molecular deformations without recurring to any elaborated formula. The proposed approach is successfully applied to analyze the experimental EXAFS data of the $\mathrm{KAu}(\mathrm{CN})_{2}$ and $\left[\mathrm{RuCl}_{2}(\mathrm{CO})_{3}\right]_{2}$ molecular complexes in which the advantages over the classical fit are demonstrated.

\section{METHODS}

The basic idea, behind our approach, stands in the construction, for each scattering process, of a signal path function $\chi_{i}$ that, under the variation of a user-defined set of $n$ structural parameters, $\boldsymbol{p}=\left(p_{1}, \ldots, p_{n}\right)$, is able to return an accurate approximation for the $i^{\text {th }}$ scattering signal. This contribution needs to be as close as possible to the one obtained through an $a b$ initio calculation involving the molecular complex affected by $p$. The choice of the structural parameters $\boldsymbol{p}$ to be refined depends exclusively on the user preference. Clearly, a certain knowledge on the system under study is required to avoid the variation of determined geometric parameters, which, rarely, can undergo towards certain kinds of deformations. A good example is represented by the double or triple bond distances connecting two atoms, which generally should be maintained almost unperturbed, as shown in the example reported in Section 3.1.

The perturbation of each element of $\boldsymbol{p}$ is reflected automatically in the change of each vector distance $\{r\}_{i}=\left(r_{1}, \ldots, r_{m}\right)$, where $r_{m}$ is the coordinate vector of the $m^{\text {th }}$ atom involved in the scattering process depending on $p$. It follows that, for a given $\chi_{i}$ path, the amplitude, the phase, and the electron mean free path can be described as nonlinear functions of $\{r(\boldsymbol{p})\}_{i}: F_{i}\left(k,\{r(\boldsymbol{p})\}_{i}\right), \phi_{i}\left(k,\{r(\boldsymbol{p})\}_{i}\right)$, and $\lambda_{i}\left(k,\{r(p)\}_{i}\right)$. These terms contribute to the path signal $\chi_{i}$, whose dependence from $\boldsymbol{p}$ can be then expressed as $\chi_{i}(k, \boldsymbol{p})$. For the sake of clarity, the variations of the scattering amplitudes and the total phase shifts for two selected SS and MS paths are showed, in correspondence of properly controlled modification of the bond distances and of the angle $\alpha$ of the $\mathrm{KAu}(\mathrm{CN})_{2}$ structure, in Figure 2 of Section 3.1.

The fitting procedure exploiting a $\mathrm{ML}$ approach can be formally divided into two parts, described in Sections 2.1 and 2.2 , respectively. First, it is necessary to identify the paths with the highest impact on the total EXAFS signal. Second, a set of nonlinear functions, approximating the dependence of each $\chi_{i}$ signal from $p$, are generated. This part foresees the training procedure of a ML algorithm. Once that all the selected $\chi_{i}(k)$ are approximated in the whole range of variation of $p$, the theoretical total EXAFS signal is generated summing over all the single contributions. Afterward, the fitting of an experimental spectrum can be performed.

2.1. Generation of a Set of ML-Based $\chi_{i}$ Functions. Supervised ML algorithms require a data set of theoretical spectra linked (i.e., labeled) to the corresponding molecule geometry. ${ }^{25,28}$ Regarding the proposed approach, only those scattering paths that contribute significantly in the selected region of the experimental spectrum are chosen for the training set generation. In the classical (geometry independent) EXAFS fit, this requirement can be satisfied considering the SS and MS paths having a curve-wave importance ratio (rank) ${ }^{6,7}$ higher than a certain user-defined value. In the case of a geometryvariable molecular complex, this step requires a well-defined methodology if the most relevant EXAFS paths are not known beforehand. Some MS paths depend strongly on the geometry 
and can become relevant for certain deformations of the molecule. For example, the gradual evolution of a collinear MS path to a triangular-one can cause the abatement of its intensity, expressed in terms of its rank. ${ }^{29}$ It follows that there could be some molecular configurations, involving the same atoms, where a MS path is significant while in the others its signal contribution is completely negligible. To consider this behavior, the following procedure is proposed.

In the range of variation of structural parameters $p$, a coarse multidimensional grid is generated (see Figure S1 in the Supporting Information). Afterward, for each point of the grid, a FEFF calculation is executed, and the rank terms associated with each path are extracted and analyzed for each deformation. Only those paths possessing a rank equal or higher than $15 \%$ and a maximum half path length $\left(R_{i}\right)$ of $5 \AA$ are selected. This approach allows considering all possible paths having a strong impact on the fitting routine independently of the starting geometry. If necessary, further user-defined filtering criteria can be introduced to limit the number of paths. For example, if a relevant path (rank > 15\%) shows for each point of the coarse grid a $R_{i}$-value higher than the fitting range, it can be properly excluded. The number of significant paths is further refined selecting only those not equivalent to each other. The term equivalent is used here to indicate two or more paths possessing the same kind of scattering atoms, showing the same $R_{i}$ and scattering angles if evaluated on the same reference molecule (i.e., the initial unperturbed). The time-reversal symmetry ${ }^{30}$ is taken into consideration too. In fact, in the case of disagreement among the scattering angles associated with two paths presenting the same $R_{i}$ and atoms type, the comparison can be repeated inverting the order of the atoms involved in one of the scattering processes. A pictorial representation of the equivalence criterion defined before is depicted in Figure S2.

All the significant, not equivalent paths obtained through this procedure will be further approximated as a function of the variation of $\boldsymbol{p}$ employing a regression approach. The latter is realized by means of an ML algorithm, which foresees a training procedure.

Herein, the multidimensional space of the configurations $\boldsymbol{P}$, generated by all combinations of the chosen geometric parameters within their range of variation (i.e., $p_{1} \times \ldots \times p_{n}$ ), is sampled uniformly according to the improved Latin hypercube sampling (IHS) method. ${ }^{31}$ This technique ensures the narrowest distance among the projections of each multidimensional point along every dimension of $\boldsymbol{P}^{25,27}$ The result is a finer multidimensional grid (in contrast to the precedent one, shown in Figure S1). For the sake of clarity, Figure S3 of the Supporting Information text shows the distribution of points employed to sample properly the space of possible configurations $\boldsymbol{P}$ associated with the example discussed in Section 3.1.

For every point of $\boldsymbol{P}$, a distorted molecular complex is generated, and a FEFF calculation is executed. This process is relatively fast. For both examples discussed in Section 3, the execution of the FEFF code for 500 input files (i.e., 500 perturbed geometries for both cases) took just few minutes. Afterward, each path, which was assumed to be relevant in the precedent step, is identified among all the FEFF scattering processes through the equivalence criterion. Herein, the path attributes, $F_{i}(k), \phi_{i}(k)$, and $\lambda_{i}(k)$, are extracted and employed to reconstruct the corresponding $\chi_{i}(k)$ signal.
At the end of this process, every not equivalent significant path possesses a ML training data set containing the corresponding path functions $\chi_{i}(k)$ evaluated for all the sampling points of $\boldsymbol{P}$. As an example, Figure S4 reports all the ML training datasets employed to generate the corresponding path functions exploited to fit the EXAFS spectrum of the $\mathrm{KAu}(\mathrm{CN})_{2}$ complex described in Section 3.1.

Starting from these data arrays, an Extra Trees regressor algorithm $^{25,27,28}$ is trained, and for each selected path, a multidimensional interpolating path function is generated: $\chi_{i}(k ; \boldsymbol{p})$. This fact implies that for any combination of the input parameters $\boldsymbol{p}=\left(p_{1}, \ldots, p_{n}\right)$, the selected path functions return as output the corresponding path signals approximated for that perturbed geometry. Finally, every path function $\chi_{i}(k ; p)$ is weighted for the related $S_{0 i}^{2}$ and $\sigma_{i}^{2}$ parameters (see eq 2) defining the approximated $\chi_{i}^{\text {approx }}$ contributions. In addition, an energy shift term $\Delta E_{0 i}$, modifying the values of $k$, can be applied to each new $\chi_{i}^{\text {approx }}$ term concluding the parametrization phase. A schematic representation of the described methodology is represented in the flow diagram reported in Figure 1.

2.2. Fitting Routine. Once all the $\chi_{i}^{\text {approx }}$ functions are generated, they can be summed together through eq 1 to obtain the theoretical EXAFS signal $\chi^{\text {THEORY }}$, which will be employed in the fitting routine. The fitting parameters are, in



Figure 1. Flow diagram reporting the procedure followed to recover the ML-derived path functions $\chi_{i}$ required to fit an arbitrary experimental EXAFS spectrum. 

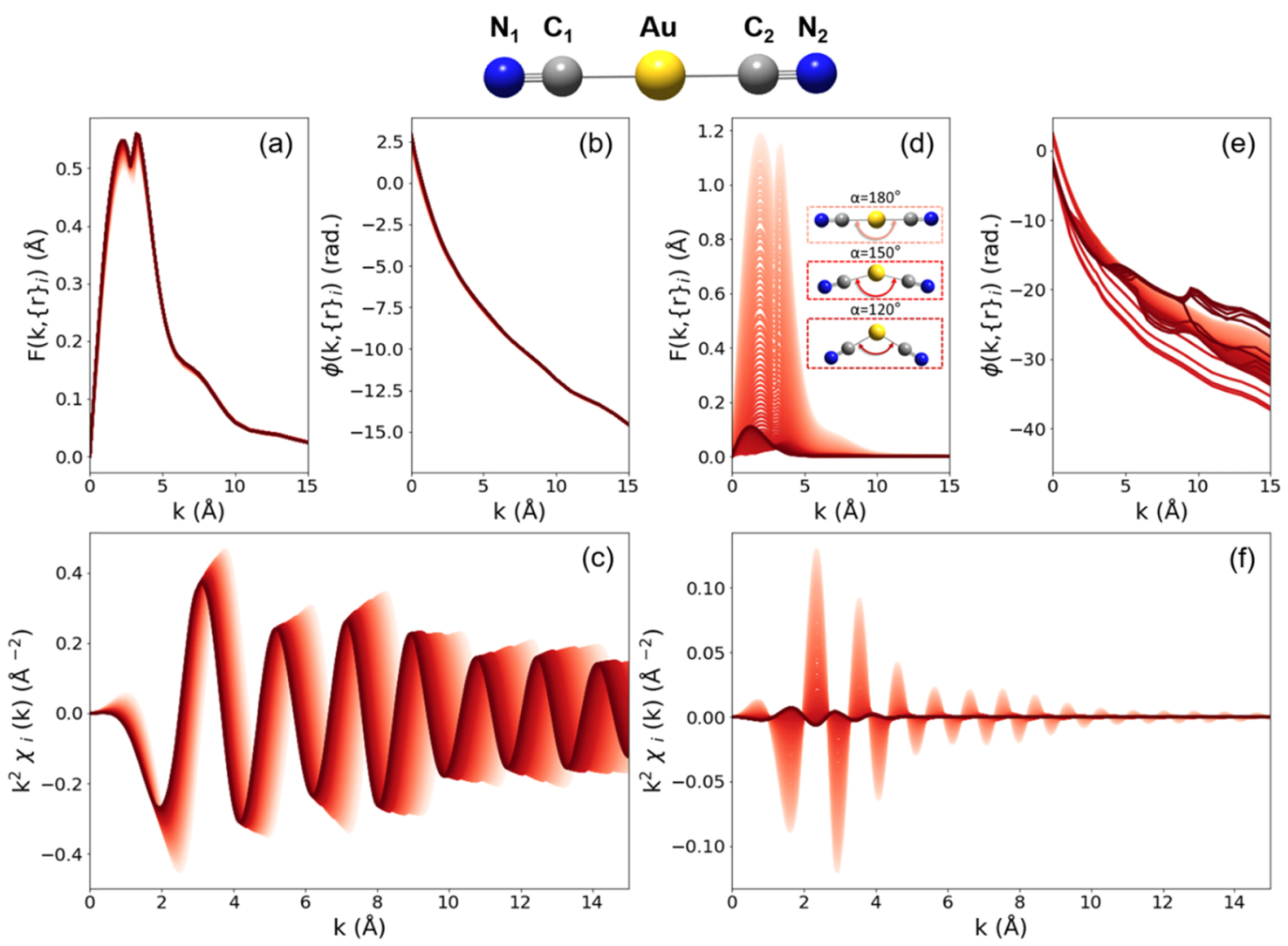

Figure 2. Effective scattering amplitudes, phase shifts, and related EXAFS contributions as a function of the deformations involving the $\mathrm{Au}(\mathrm{CN})_{2}{ }^{-}$ molecule showed on the top of the figure. Panels (a), (b), and (c) refer to a single scattering process referring to the paths $\mathrm{Au} \rightarrow \mathrm{C}_{1} \rightarrow \mathrm{Au}$ or $\mathrm{Au} \rightarrow$ $\mathrm{C}_{2} \rightarrow \mathrm{Au}$. The curves have been generated by shifting in parallel the $\mathrm{C}_{1} \mathrm{~N}_{1}$ and $\mathrm{C}_{2} \mathrm{~N}_{2}$ groups along the bond directions from $-0.12 \AA$ (light red curve) to $+0.12 \AA$ (dark red curve) with respect to the crystallographic structure. Panels (d), (e), and (f) are related to the three-leg MS paths: Au $\rightarrow \mathrm{C}_{1} \rightarrow \mathrm{C}_{2} \rightarrow \mathrm{Au}$ or $\mathrm{Au} \rightarrow \mathrm{C}_{2} \rightarrow \mathrm{C}_{1} \rightarrow \mathrm{Au}$, which change from a collinear configuration $\left(\alpha=180^{\circ}\right.$ : light red curve $)$ to a triangular $\left(\alpha=120^{\circ}:\right.$ dark red curve). Color code: Au (yellow), N (blue), and C (gray).

this case, $\left(p_{1}, \ldots, p_{n}\right)$, common to every $\chi_{i}^{\text {approx }}$ function and $\left(S_{0 i}^{2}\right.$ $\sigma_{i}^{2}$, and $\left.\Delta E_{0 i}\right)$, which can assume different values and parameterizations on the basis of the number and type of atoms involved in the selected paths.

In this work, the fit of the experimental EXAFS signal in the direct $(R)$ space was performed minimizing the following $\mathrm{L}_{2}$ norm function:

$$
\Xi(R)=\frac{\sum_{i}\left\|\Delta \hat{\chi}(R)_{i}\right\|^{2}}{\sum_{i}\left\|\hat{\chi}^{\text {DATA }}(R)_{i}\right\|^{2}}
$$

Where $\Delta \hat{\chi}(R)$ is the complex residual function defined as:

$$
\begin{aligned}
\Delta \hat{\chi}(R)= & \Re\left[\hat{\chi}^{\text {DATA }}(R)_{i}-\hat{\chi}^{\text {THEORY }}(R)_{i}\right] \\
& +\Im\left[\hat{\chi}^{\text {DATA }}(R)_{i}-\hat{\chi}^{\text {THEORY }}(R)_{i}\right]
\end{aligned}
$$

while $\|\ldots\|^{2}$ is the Euclidean norm. The $\hat{\chi}^{\text {DATA }}$ and $\hat{\chi}^{\text {THEORY }}$ terms represent the Fourier transforms of the experimental and theoretical EXAFS signals weighted for an arbitrary $k^{n}$ factor.

The minimization of $\Xi$ can be realized employing the standard least-squares approach, ${ }^{32}$ the simplex method, ${ }^{33}$ or even a branch of different other techniques, implemented, for example, in the MINUIT CERN library. ${ }^{34}$ In the proposed method, since the terms $\chi_{i}^{\text {approx }}$ are noncontinuous numerical functions derived by the Extra Trees approximation, the Powell algorithm, ${ }^{35,36}$ not requiring any derivatives of eq 3, was used for the minimization routine. The ML-based path functions return, for any combination of parameters, the corresponding path signal almost immediately. This fact makes the time required for the minimization of the objective $\left(\mathrm{L}_{2}\right.$ norm) function through this algorithm almost comparable with the one required by the Artemis code.

The parameter uncertainties can be estimated in various ways. Usually, the main strategy consists of considering the inverse of the Hessian $(\mathrm{H})$ of $\Xi$ evaluated in correspondence with its minimum value. It is possible to demonstrate, in fact, that the diagonal elements of $\mathrm{H}^{-1}$ are the squared parameter errors while the off-diagonal elements, when divided by the square root of the product of the corresponding diagonal elements, provide the related correlation values. ${ }^{37,38}$ However, this approach may fail when the minimum of eq 3 is close to a boundary $^{39}$ or when the target function $\Xi$ is nondifferentiable, as in this case. Herein, we estimated the uncertainties in the form of statistical test recurring to the Fisher (F) test. ${ }^{40}$ Once a global minimum is found, for each section of $\Xi$, it is possible to vary the related parameter in the neighbor of the minimum and evaluate the region where the $\mathrm{F}$ variable increases above a userdefined confidence level (here set to $68 \%)^{13,41}$ assuming a number of degree of freedom (D.O.F) equal to: $N_{\text {idp }}-\nu$, where $\nu$ is the number of fitting parameters. Further information about this statistical approach can be found in refs 42, 43.

Finally, a more sophisticated fitting procedure involving the wavelet transform $(\mathrm{WT})^{44}$ representation has also been 
implemented and tested although no considerable differences emerged using this space of fit. The details of this last fitting procedure are reported in Section S5 of the Supporting Information, while for the sake of completeness the WT fit of the EXAFS spectrum referring to $\left[\mathrm{RuCl}_{2}(\mathrm{CO})_{3}\right]_{2}$ has been added to the final part of Section 3.2. The entire methodology described in this manuscript was developed in Python using the ML libraries of the PyFitIt code.

\section{RESULTS AND DISCUSSION}

3.1. Case Study for the $\mathrm{KAu}(\mathrm{CN})_{2}$ Complex. In this section, we present, as a first case study, the ML-based EXAFS analysis of the $\mathrm{KAu}(\mathrm{CN})_{2}$ molecular complex illustrated in the top panel of Figure 2. Details concerning the data acquisition techniques are reported in Section S2 of the Supporting Information. The dicyanoaurate ion $\mathrm{Au}(\mathrm{CN})_{2}{ }^{-}$is a tecton, widely employed in solid-state chemistry because of the versatility of the intermolecular interactions, consisting of hydrogen bonds and aurophilic contacts that it can establish. ${ }^{4-50}$ In addition, its linear shape favors the formation of supramolecular 1D, 2D, and 3D networks that have demonstrated interesting properties of phosphorescence, vapochromism, and giant negative linear compressibility. ${ }^{48,51-55}$ The structure is characterized by an approximatively linear geometry.

Starting from the crystallographic $\mathrm{Au}(\mathrm{CN})_{2}{ }^{-}$structure, ${ }^{48}$ three structural parameters have been selected for the refinement (see Figure S5): the two $\mathrm{Au}-(\mathrm{CN})$ distances $\Delta d_{\mathrm{Au}-(\mathrm{CN})}$, the variation $\Delta \alpha$ of the angles defined by the rotation of the bond axis $\mathrm{Au}-\mathrm{C}_{1} \mathrm{~N}_{1}$ and $\mathrm{Au}-\mathrm{C}_{2} \mathrm{~N}_{2}$, and finally the tilting $\Delta \beta$ of the $\mathrm{C}_{1}-\mathrm{N}_{1}$ and $\mathrm{C}_{2}-\mathrm{N}_{2}$ bonds. The $\mathrm{C}-\mathrm{N}$ distances were maintained fixed at a crystallographic value of $1.174 \AA$. The coarse grid associated with these parameters was generated assuming a range of variation for $\Delta d_{\mathrm{Au}-(\mathrm{CN})}$ within -0.12 and $+0.12 \AA$ and for $\Delta \alpha$ and $\Delta \beta$ within $-15^{\circ}$ and $+30^{\circ}$ with respect to the initial structure.

Based on the FEFF calculations, small variations are observed in the amplitudes and phases of the SS paths involving the $\mathrm{Au}$ absorber and the $\mathrm{C}_{1}$ and $\mathrm{C}_{2}$ scattering atoms (Figure $2 \mathrm{a}-\mathrm{c}$ ) as a function of the $\mathrm{Au}-\mathrm{C}$ bond distances. On the other hand, an abrupt diminution of the amplitude and a nonlinear variation of the phase were found for the MS paths $\mathrm{Au} \rightarrow \mathrm{C}_{1} \rightarrow \mathrm{C}_{2} \rightarrow \mathrm{Au}$ (or $\mathrm{Au} \rightarrow \mathrm{C}_{2} \rightarrow \mathrm{C}_{1} \rightarrow \mathrm{Au}$ ) varying the angle $\alpha$ from 180 to $120^{\circ}$ (Figure $2 \mathrm{~d}-\mathrm{f}$ ). To select the most relevant paths required for the fitting procedure (see Section 2.1 ), we fixed the rank cutoff at $15 \%$. Under this constraint, the list of the most intense not equivalent paths contained the following items: $\mathrm{Au} \rightarrow \mathrm{C}_{1} \rightarrow \mathrm{Au}$ (SS), $\mathrm{Au} \rightarrow \mathrm{N}_{1} \rightarrow \mathrm{Au}$ (SS), $\mathrm{Au} \rightarrow \mathrm{C}_{1} \rightarrow \mathrm{N}_{1} \rightarrow \mathrm{Au}$ (MS with three legs), $\mathrm{Au} \rightarrow \mathrm{C}_{1} \rightarrow \mathrm{N}_{1}$ $\rightarrow \mathrm{C}_{1} \rightarrow \mathrm{Au}$ (MS with four legs), and finally $\mathrm{Au} \rightarrow \mathrm{C}_{1} \rightarrow \mathrm{Au}$ $\rightarrow \mathrm{C}_{2} \rightarrow \mathrm{Au}$ (MS with four legs).

In general, because of the not absolute symmetry of the $\mathrm{CN}$ groups with respect to the $\mathrm{Au}$ absorber (in terms of bond distances), we noted that for some perturbed geometry, the FEFF pathfinder provides the path $\mathrm{Au} \rightarrow \mathrm{C}_{2} \rightarrow \mathrm{Au}$ with degeneration 2 instead of $\mathrm{Au} \rightarrow \mathrm{C}_{1} \rightarrow \mathrm{Au}$. Independent of the atoms enumeration, these paths were considered under the same typology: $\mathrm{Au} \rightarrow \mathrm{C}_{1} \rightarrow \mathrm{Au}$. This approach was extended also to every relevant MS path in the case of disagreement with the ones indicated in the not equivalent path list. The finer grid in the space of structural parameters was built varying the quantities $\Delta d_{\mathrm{Au}-(\mathrm{CN})}, \Delta \alpha$, and $\Delta \beta$ for 500 times following the IHS scheme. Afterward, for each selected deformation, the related input structure was generated and the FEFF routine was executed.

In the case of two (or more) equivalent paths for the same molecular deformation, for example, $\mathrm{Au} \rightarrow \mathrm{C}_{1} \rightarrow \mathrm{Au}$ and $\mathrm{Au}$ $\rightarrow \mathrm{C}_{2} \rightarrow \mathrm{Au}$ with degeneration 1 , both the signals were extracted, summed, and saved in the corresponding data set named, in this case $\mathrm{Au} \rightarrow \mathrm{C}_{1} \rightarrow \mathrm{Au}$. This approach can be clearly followed, if and only if, it is supposed that both the paths are characterized by the same DW parameter. If this condition is not satisfied (not considered in this example), the $\mathrm{Au} \rightarrow \mathrm{C}_{2} \rightarrow \mathrm{Au}$ must be decoupled from the $\mathrm{Au} \rightarrow \mathrm{C}_{1} \rightarrow \mathrm{Au}$ path and considered as a separate contribution.

At the end of the selection procedure, five training datasets $\left(\mathrm{Au} \rightarrow \mathrm{C}_{1} \rightarrow \mathrm{Au}, \mathrm{Au} \rightarrow \mathrm{N}_{1} \rightarrow \mathrm{Au}, \mathrm{Au} \rightarrow \mathrm{C}_{1} \rightarrow \mathrm{N}_{1} \rightarrow \mathrm{Au}, \mathrm{Au}\right.$ $\rightarrow \mathrm{C}_{1} \rightarrow \mathrm{N}_{1} \rightarrow \mathrm{C}_{1} \rightarrow \mathrm{Au}$, and $\left.\mathrm{Au} \rightarrow \mathrm{C}_{1} \rightarrow \mathrm{Au} \rightarrow \mathrm{C}_{2} \rightarrow \mathrm{Au}\right)$ were employed to train the regressor algorithm. The quality of prediction associated with each nonlinear path function was obtained recurring to the 10 -fold cross-validation (CV) technique. ${ }^{25}$ The $\mathrm{CV}$ results, reported in Table $\mathrm{S} 1$, indicate also here an optimal reproducibility for each not equivalent path supposed to characterize the complex. The DW factors associated with the SS paths, $\mathrm{Au} \rightarrow \mathrm{C}_{1} \rightarrow \mathrm{Au}$ and $\mathrm{Au} \rightarrow \mathrm{N}_{1} \rightarrow$ $\mathrm{Au}$, were defined using the quantities $\sigma_{\mathrm{C}}^{2}$ and $\sigma_{\mathrm{N}}^{2}$. On the other hand, the MS DW terms were parametrized as: $\sigma_{\mathrm{MS}}^{2}=\sqrt{\sum_{i} \sigma_{i}^{2}}$, where $\sigma_{i}^{2}$ are the DW factors of the atoms involved in the scattering process. The latter constitutes the unique parameterization required by this method. For all the scattering paths, common $S_{0}^{2}$ and $\Delta E_{0}$ were assumed.

The fit of the EXAFS spectrum was carried out in the $R$ space range from 1 up to $4 \AA$, assuming a $k$-space range for the Fourier transform within 2.6 and $12 \AA^{-1}$, resulting in the number of independent points $N_{\mathrm{idp}} \approx 18$. Seven fitting parameters were employed in the refinement: $\Delta d_{\mathrm{Au}-(\mathrm{CN})}, \Delta \alpha$, $\Delta \beta, \sigma_{\mathrm{C}}^{2}, \sigma_{\mathrm{N}}^{2}, S_{0}^{2}$, and $\Delta E_{0}$. The EXAFS fit was realized inside their related range of variation. In addition to the $\Delta d_{\mathrm{Au}-(\mathrm{CN})}$, $\Delta \alpha$, and $\Delta \beta$ described above, the following further bounds were considered: $\sigma_{C}^{2}$ and $\sigma_{\mathrm{N}}^{2} \in(0.001 \div 0.015) \AA^{2}, S_{0}^{2} \in(0.8$ $\div 1.3)$, and $\Delta E_{0} \in(-10 \div 10) \mathrm{eV}$. The result of the minimization of eq 3 is summarized in Table 1 together with

Table 1. Best-Fit Parameters ( $\mathrm{L}_{2}$ Minimization Output) Obtained Minimizing the Target Function of Eq 3 Considering the Set of Deformations Shown in Figure $S 5^{a}$

\begin{tabular}{llc}
\multicolumn{2}{c}{ best-fit parameters } & confidence intervals \\
$S_{0}^{2}$ & 0.91 & $0.8 \div 1.04$ \\
$\Delta E_{0}(\mathrm{eV})$ & 7.92 & $6.38 \div 9.71$ \\
$\sigma_{\mathrm{C}}^{2}\left(\AA^{2}\right)$ & 0.0024 & $0.001 \div 0.005$ \\
$\sigma_{\mathrm{N}}^{2}\left(\AA^{2}\right)$ & 0.001 & $0.001 \div 0.004$ \\
$\Delta d_{\mathrm{Au}-(\mathrm{CN})}(\AA)$ & -0.01 & $-0.03 \div 0.01$ \\
$\alpha\left({ }^{\circ}\right)$ & 168.88 & $138.84 \div 210$ \\
$\beta\left({ }^{\circ}\right)$ & -1.32 & $-15 \div 11.42$
\end{tabular}

${ }^{a_{T}}$ The uncertainties reported in the last column were obtained through the F-test fixing a confidence level of $68 \%$ for 11 D.O.F.

the parameter confidence intervals, while the comparison between the experimental and best-fit FT EXAFS representations is reported in Figure 3. The fit of the EXAFS spectrum employing the WT representation is shown in Section S5.1 of the Supporting Information. The best-fit results obtained with this approach have been further verified through an EXAFS fit realized using the Artemis software. Herein, the refined 


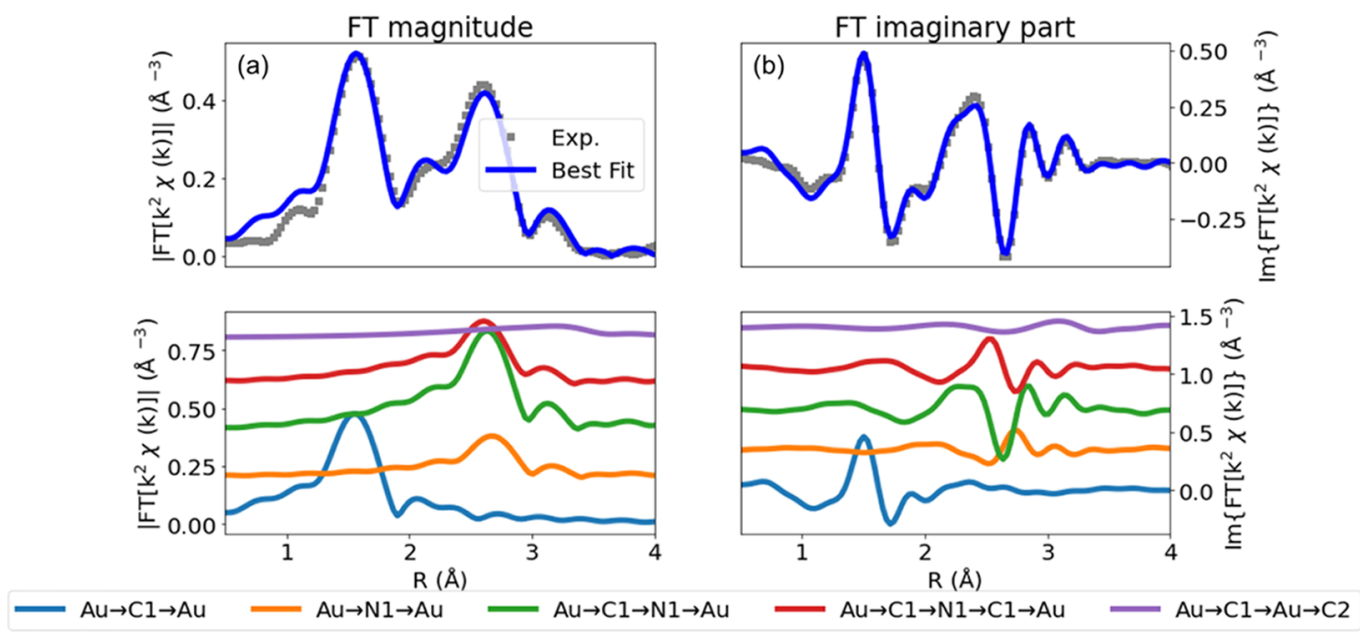

Figure 3. Phase-uncorrected (a) modulus and (b) imaginary part of the experimental and best-fit FT EXAFS spectra for the KAu(CN $)_{2}$ complex. The experimental data are shown as gray squares while the best-fits with blue solid lines. The principal SS and MS contributions included in the fitting model are reported in the bottom panels as colored solid lines, vertically translated for the sake of the clarity.
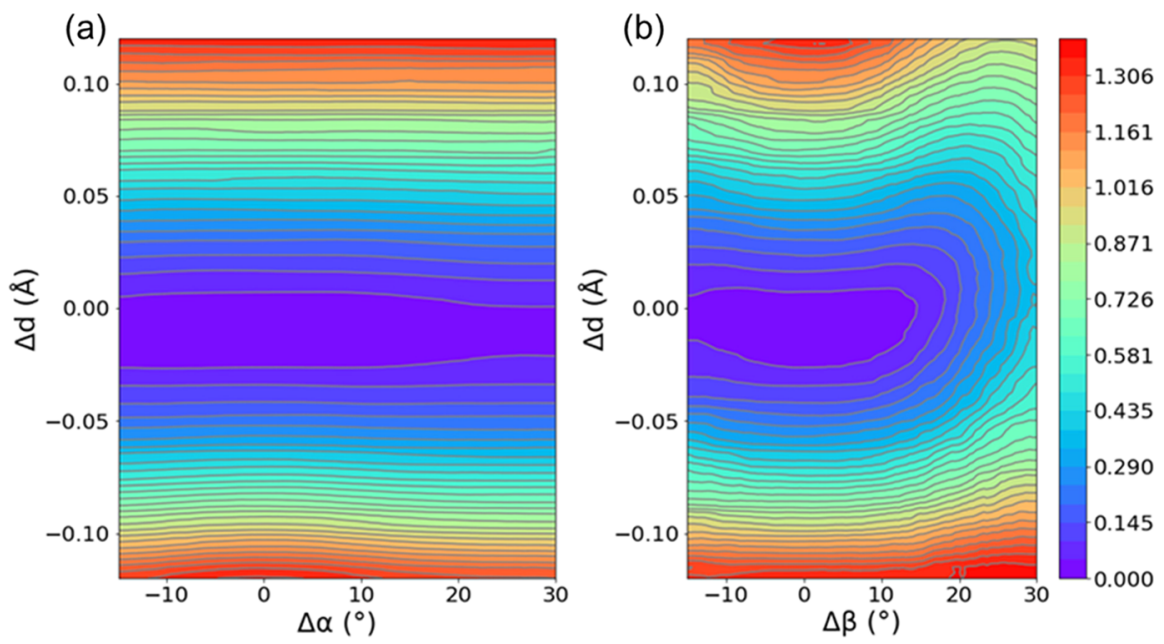

Figure 4. Contour plot map of $\Xi$ obtained varying and $\Delta \alpha$ maintaining all the remaining parameters fixed to the best-fit values. (b) Same as (a) but varying $\Delta \beta$.

parameters were used to generate the corresponding KAu$(\mathrm{CN})_{2}$ structure that has been employed in the least-squares optimization, presented in Section S6.1 of the S.I text. The latter shows a $\% R$-factor value (vide infra) identical to the one found by our approach, and the deviations from the MLrefined parameters are comparable to zero.

Figure 3 demonstrates that all the main spectral features, because of SS and MS effects, appearing in the experimental FT EXAFS spectrum are properly reproduced by the best-fit curve. The $\% R$-factor (IXS) ${ }^{56}$ associated with the fit is found to be equal to $1.3 \%$, showing that the theoretical representation is in good agreement with the experiment. The small misfit between the theoretical and the experimental EXAFS curves, particularly visible in the resulting FT-magnitude in Figure 3a at ca. $2.5-2.75 \AA$, is attributed to low-intensity SS and MS contributions because of the presence of water molecules surrounding the gold complex, neglected in this example.

The $\Delta d_{\mathrm{Au}-(\mathrm{CN})}$ quantity is refined to $-0.01 \AA$, which is a negligible contraction of the $\mathrm{CN}$ groups from the $\mathrm{Au}$ absorber, in line with the crystallographic model. The variation of $\Delta \alpha$ from the initial configuration is not found to be equal to $0^{\circ}$ (i.e., $\alpha=180^{\circ}$ ) but of ca. $5.57^{\circ}$ (i.e., $\alpha \approx 169^{\circ}$ ). However, the uncertainty associated with the estimation of this parameter is quite large. Thanks to the ML-based approach, it is possible to analyze in fact the $\Delta \alpha$ dependence of the $\Xi$ section, as shown in Figure S6a. It is evident that the $\Xi$ function shows a weak variation as a function of $\Delta \alpha$. In particular, the $\Xi$ trend does not possess an appropriate unique minimum but a flat line extending within ca. $\pm 7.2^{\circ}\left(\alpha \approx 194.4^{\circ}\right)$ and $\left(\alpha \approx 165.6^{\circ}\right)$. Furthermore, it is worth noting that the $\mathrm{Au} \rightarrow \mathrm{C}_{1} \rightarrow \mathrm{Au} \rightarrow \mathrm{C}_{2}$ $\rightarrow \mathrm{Au}$ path is the only EXAFS contribution possessing a connection with the $\Delta \alpha$ variation. If compared with all the other not equivalent scattering processes affecting the total EXAFS signal, it possesses always the lowest rank intensity for every kind of selected deformation as shown in Section S3.3. This fact shows that the influence of $\Delta \alpha$ on $\Xi$ is overpowered by the other parameters involved in the refinement, see Figure 4a. Despite this fact, the exclusion of the $\mathrm{Au} \rightarrow \mathrm{C}_{1} \rightarrow \mathrm{Au} \rightarrow \mathrm{C}_{2}$ $\rightarrow \mathrm{Au}$ path from the fitting procedure causes an increase of the $\% R$-factor to ca. $2.4 \%$, indicating the necessity of its employment.

Most of the confidence intervals for the fitted parameters, shown in Table 1, are not symmetric. This evidence is principally because of the employment of boundaries regions 

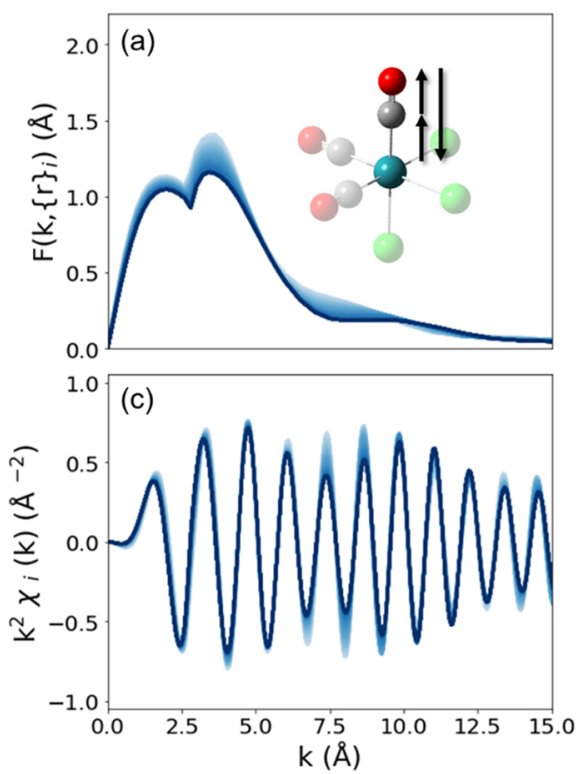
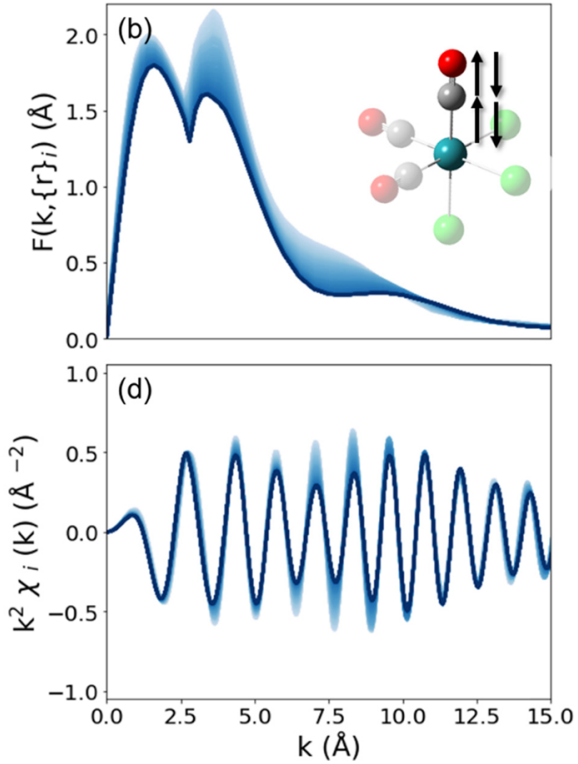

Figure 5. Trend of the backscattering amplitudes (on the top) and of the related EXAFS path function (on the bottom) referring to the Ru $\rightarrow \mathrm{C}_{1}$ $\rightarrow \mathrm{O}_{1} \rightarrow \mathrm{Ru}(\mathrm{a}, \mathrm{c})$ and $\mathrm{Ru} \rightarrow \mathrm{C}_{1} \rightarrow \mathrm{O}_{1} \rightarrow \mathrm{C}_{1} \rightarrow \mathrm{Ru}(\mathrm{b}, \mathrm{d})$ scattering process showed in the insets, respectively. The curves have been obtained keeping fixed the $\mathrm{Ru}-\mathrm{O}$ distance at $3 \AA$ and varying the $\mathrm{Ru}-\mathrm{C}$ bond length from $1.78 \AA$ (light blue) to $2.02 \AA$ (dark blue). Color code: $\mathrm{Ru}$ (blue), $\mathrm{Cl}$ (green), $\mathrm{O}$ (red), and C (gray).

during the minimization routine. An interesting result is represented by the $\mathrm{DW}$ value for the $\mathrm{SS} \mathrm{Au} \rightarrow \mathrm{N}$ path, which coincides with the lowest boundary of the $\sigma_{\mathrm{N}}^{2}$ range of variation. This result can be understood considering that the DW factor is a measure of the mean-square displacement of the atoms from their equilibrium position. In the analyzed example, each $\mathrm{N}$ atom is connected to a $\mathrm{C}$ atom through a triple bond avoiding its independent variation, justifying this finding.

Considering the $\Delta \beta$ parameter (connected to the $\mathrm{Au} \rightarrow \mathrm{C}_{1}$ $\rightarrow \mathrm{N}_{1} \rightarrow \mathrm{Au}$ and $\mathrm{Au} \rightarrow \mathrm{C}_{1} \rightarrow \mathrm{N}_{1} \rightarrow \mathrm{C}_{1} \rightarrow \mathrm{Au}$ MS paths), its variation is assessed to be ca. $-1.3^{\circ}$. However, as for the precedent case, the related confidence interval suggests that this value is comparable with the null variation of $\Delta \beta$. In particular, $\Xi$ shows a negligible variation for the $\beta$ values within ca. -6.3 and $6.3^{\circ}$, as shown in Figure S6b. Below and above these two quantities, the changes of $\Xi$ are more intense than for the $\alpha$ case determining a narrower uncertainty range. This fact is also evident analyzing the contour plot of $\Xi$ evaluated for $\Delta d_{\mathrm{Au}-(\mathrm{CN})}$ and $\Delta \beta$, see Figure $4 \mathrm{~b}$, where a more localized valley can be clearly identified.

Summarizing this first case study, we demonstrated that it is possible to obtain a detailed description of the bond distances through the EXAFS fitting procedure, validating the initial unperturbed model. Moreover, the ML approach allowed investigating the angular dependence of the principal MS paths emerging from the selected structure. Herein, we have showed that these contributions are approximatively stable for small variations of the related $\alpha$ and $\beta$ angles around the initial (linear) structure determining an enlargement of the uncertainty associated with their estimation through the EXAFS fitting procedure, while for their larger perturbations, stronger changes in $\Xi$ appear connected to an abrupt diminution of the reproduction of the experimental spectrum using the theoretical one.

3.2. Case Study for the $\left[\mathrm{RuCl}_{2}(\mathrm{CO})_{3}\right]_{2}$ Complex. Halogenocarbonylruthenium complexes have a range of promising catalytic ${ }^{57,58}$ and biomedical ${ }^{58-60}$ applications. In this section, we analyze the EXAFS data of the $\left[\mathrm{RuCl}_{2}(\mathrm{CO})_{3}\right]_{2}$ complex in which the so-called forward MS paths have a strong dependence on the position of the intermediate scatterer (vide infra). The experimental details and data collection were recently reported by De Vos and coworkers. ${ }^{58}$ Because the $\mathrm{Ru}-\mathrm{Ru}$ contribution is negligible, the binuclear structure of $\left[\mathrm{RuCl}_{2}(\mathrm{CO})_{3}\right]_{2}$ was approximated by a mononuclear $\mathrm{RuCl}_{3}(\mathrm{CO})_{3}$ model (molecular structure shown in Figure $5 a)$. Without changing the angle with respect to the initial $D_{4 h}$ symmetry, only the three interatomic distances, $\mathrm{Ru}-\mathrm{O}\left(\Delta d_{\mathrm{O}}\right)$, $\mathrm{Ru}-\mathrm{C}\left(\Delta d_{\mathrm{C}}\right)$, and $\mathrm{Ru}-\mathrm{Cl}\left(\Delta d_{\mathrm{Cl}}\right)$, were varied (see Figure S8) within the -0.12 to $0.12 \AA$ range with respect to the starting geometry. With a rank cutoff set to $15 \%$, the following paths were selected: $\mathrm{Ru} \rightarrow \mathrm{C}_{1} \rightarrow \mathrm{Ru}$ (SS), $\mathrm{Ru} \rightarrow \mathrm{O}_{1} \rightarrow \mathrm{Ru}$ (SS), $\mathrm{Ru}$ $\rightarrow \mathrm{Cl}_{1} \rightarrow \mathrm{Ru}$ (SS), $\mathrm{Ru} \rightarrow \mathrm{O}_{1} \rightarrow \mathrm{C}_{1} \rightarrow \mathrm{Ru}$ (MS with three legs), and finally $\mathrm{Ru} \rightarrow \mathrm{C}_{1} \rightarrow \mathrm{O}_{1} \rightarrow \mathrm{C}_{1} \rightarrow \mathrm{Ru}$ (MS with four legs). It is important to note that for the single-forward ( $\mathrm{Ru} \rightarrow$ $\mathrm{C} \rightarrow \mathrm{O} \rightarrow \mathrm{Ru})$ and double-forward $(\mathrm{Ru} \rightarrow \mathrm{C} \rightarrow \mathrm{O} \rightarrow \mathrm{C} \rightarrow$ $\mathrm{Ru}) \mathrm{MS}$ paths, a strong dependence of the scattering amplitude on the position of the intermediate $\mathrm{C}$ atom is observed even if the total path length (i.e., $\mathrm{Ru}-\mathrm{O}$ distance) is fixed, as demonstrated in Figure 5. This fact significantly influences the fit quality within a classical procedure as demonstrated in Section 3.3.

As in the previous example, the finer grid was built varying the $\mathrm{Ru}-\mathrm{O}, \mathrm{Ru}-\mathrm{C}$, and $\mathrm{Ru}-\mathrm{Cl}$ distances 500 times following the IHS scheme and selecting all not equivalent paths after the FEFF routine. The 10 -fold CV results, reported in Table S2, indicate an optimal reproducibility for each selected not equivalent path.

The five nonlinear path functions were then weighted for their related DW factors. The DW factors associated with the SS paths, $\mathrm{Ru} \rightarrow \mathrm{C}_{1} \rightarrow \mathrm{Ru}, \mathrm{Ru} \rightarrow \mathrm{O}_{1} \rightarrow \mathrm{Ru}$, and $\mathrm{Ru} \rightarrow \mathrm{Cl}_{1} \rightarrow$ $\mathrm{Ru}$, were defined using the related quantities: $\sigma_{\mathrm{C}}^{2}, \sigma_{\mathrm{O}}^{2}$, and $\sigma_{\mathrm{Cl}}^{2}$. On the other hand, the MS DW terms have been parametrized with the same $\sigma^{2}$ o term. For all the paths, the variables $S_{0}^{2}$ and 
$\Delta E_{0}$ were assumed common. The theoretical and experimental EXAFS signals were Fourier-transformed in the 4-16 $\AA^{-1}$ range while the fit was performed in the $R$ range from 1 to $3 \AA$ $\left(N_{\text {idp }} \approx 15\right)$. The target function shown in eq 3 is characterized, in this case, by eight fitting parameters: $\mathrm{Ru}-$ $\mathrm{O}, \mathrm{Ru}-\mathrm{C}$, and $\mathrm{Ru}-\mathrm{Cl}$ distances, $\sigma_{\mathrm{C}}^{2}, \sigma_{\mathrm{O}}^{2}$, and $\sigma_{\mathrm{Cl}}^{2}, S_{0}^{2}$, and $\Delta E_{0}$, with the boundary conditions $\sigma_{i}^{2} \in(0.001 \div 0.01) \AA^{2}$ for all DW factors, $S_{0}^{2} \in(0.8 \div 1.3)$, and $\Delta E_{0} \in(-10 \div 10) \mathrm{eV}$. The fit results are shown in Table 2 together with the parameter

Table 2. Best-Fit Parameters ( $\mathrm{L}_{2}$ Minimization Output) Obtained Minimizing the Target Function of eq 3 Considering the Set of Deformations Reported in Figure S9 ${ }^{a}$

\begin{tabular}{lll}
\multicolumn{2}{c}{ best-fit parameters } & confidence intervals \\
$S_{0}^{2}$ & 1.21 & $0.93 \div 1.3$ \\
$\Delta E_{0}(\mathrm{eV})$ & -2.06 & $-5.68 \div 1.07$ \\
$\sigma_{\mathrm{C}}^{2}\left(\AA^{2}\right)$ & 0.004 & $0.0015 \div 0.008$ \\
$\sigma_{\mathrm{O}}^{2}\left(\AA^{2}\right)$ & 0.0055 & $0.0029 \div 0.009$ \\
$\sigma_{\mathrm{Cl}}\left(\AA^{2}\right)$ & 0.0048 & $0.0027 \div 0.0075$ \\
$\mathrm{Ru}-\mathrm{C}(\AA)$ & 1.91 & $1.89 \div 1.94$ \\
$\mathrm{Ru}-\mathrm{O}(\AA)$ & 3.034 & $3.003 \div 3.067$ \\
$\mathrm{Ru}-\mathrm{Cl}(\AA)$ & 2.41 & $2.38 \div 2.43$
\end{tabular}

${ }^{a}$ On the right columns are indicated the parameter uncertainties obtained through the F-test approach fixing a confidence level of $68 \%$ for 7 D.O.F.

confidence intervals. The best-fit FT EXAFS curves are reported in Figure 6. The \%R-factor (IXS) ${ }^{56}$ is $0.9 \%$, demonstrating that the theoretical representation is in good agreement with the experiment in the selected range of fit. Similarly, to what has been done in Section 3.1, we reinforced our finding fitting with the Artemis software the ML-refined structure; see Section S6.2 of the Supporting Information. Also, for this case study, the deviations of the structural parameters from the ML-refined ones are close to zero, and the $\% R$-factor remains stable to $0.9 \%$, underlying the goodness of our results.

As a proof of concept of the results obtained through the classical FT EXAFS fit, we show, in the following, also the refinement of the above structure employing the WT representation. Details concerning the target function definition are reported in Section S5 of the Supporting Information. The comparison between the theoretical and experimental WT 2D maps is reported in Figure 7, while the best-fit parameters obtained though the WT refinement are indicated in Table 3. As it is possible to see from the comparison, all the main WT features of the experimental spectrum are properly reproduced by the theoretical WT representation. The $R$-factor associated with the fit is found to be equal to the one proper of the FT-based fit, while the confidence intervals, evaluated for each parameter involved in the fit, are close to the ones shown in Table 2.

Summarizing this case study, it is important to note that an example with the strong dependence of the SS paths on the position of the intermediate atoms was considered, and the advantage of the developed ML-based fit of EXAFS over classical fit was demonstrated.

3.3. Fit of the $\left[\mathrm{RuCl}_{2}(\mathrm{CO})_{3}\right]_{2}$ Complex Using the Classic EXAFS Fit: When the Initial Geometry Makes the Difference. As demonstrated in Figure 5, two MS paths which provide an evident contribution to the EXAFS signal have a strong dependency on the input geometry. Below, the standard EXAFS fit using Artemis software was performed.

First, the initial geometry was characterized by $\mathrm{Ru}-\mathrm{C}$ and $\mathrm{Ru}-\mathrm{O}$ distances of 1.70 and $3.0 \AA$, respectively. The fit, reported in Figure 8a, using $k^{2}$-weighted data in the $4-16 \AA^{-1}$ range results in a $\% R$-factor of ca. $0.7 \%$. The fitted $R_{\mathrm{Ru}-\mathrm{C}}$ is $1.90 \pm 0.01 \AA$ while the $R_{\mathrm{Ru}-\mathrm{O}}$ is found to be equal to $3.04 \pm$ $0.01 \AA ̊$.

It is possible to see that the $\% R$-factor is slightly lower than the one obtained using the ML-fitting approach; however, it is possible to demonstrate easily that the fitting result obtained by the Artemis code appears to depend on the input geometry, allowing to accommodate just a small variation of distances starting from the guessed structure. This fit requires, in fact, a considerable shift of the $\mathrm{Ru}-\mathrm{C}$ distance from $1.70 \AA$ to the best-fit value of $1.90 \AA$. Correcting the $\mathrm{RuCl}_{3}(\mathrm{CO})_{3}$ structure on the basis of this result and performing again the fit, it is possible to observe that the $\mathrm{Ru}-\mathrm{C}$ distance is refined by the least-squares approach ${ }^{32}$ with a negligible variation of $\Delta d_{\mathrm{C}}=$

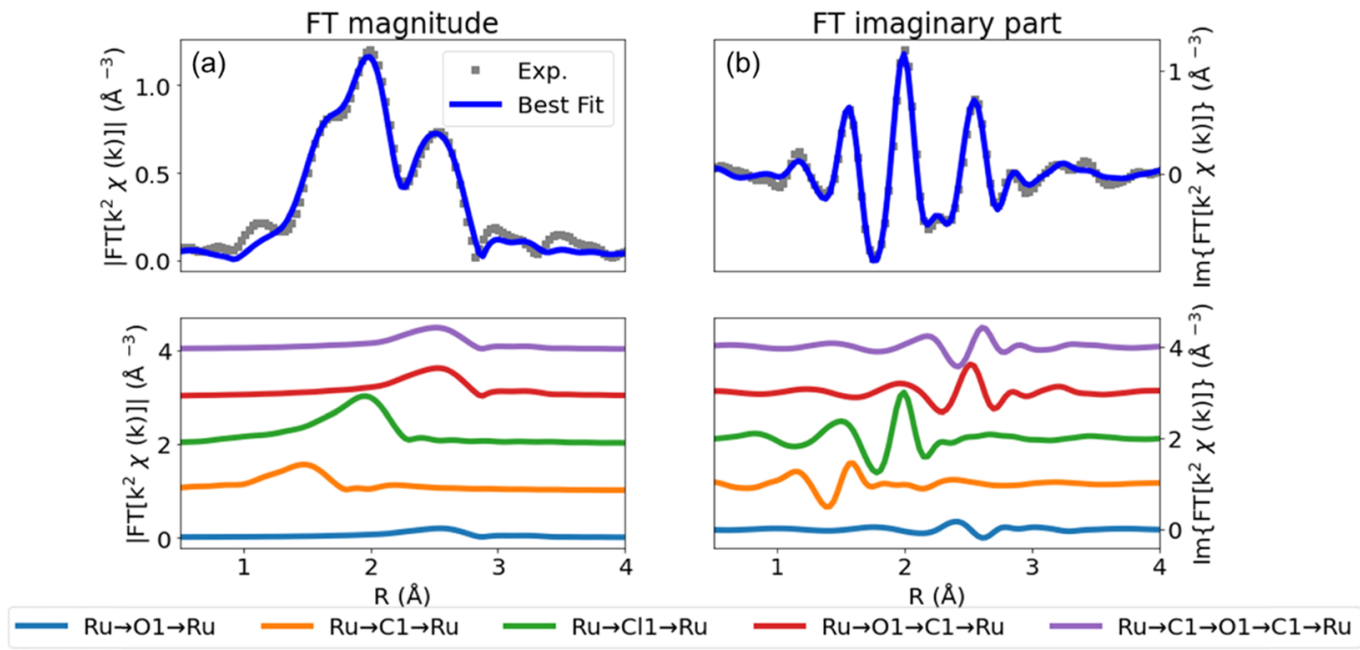

Figure 6. Phase-uncorrected (a) modulus and (b) imaginary part of the experimental and best-fit FT EXAFS spectra for $\left[\mathrm{RuCl}{ }_{2}(\mathrm{CO})_{3}\right]_{2} \cdot \mathrm{The}$ experimental data are shown as gray squares while the best-fits with blue solid lines. The principal SS and MS contributions included in the fitting model are reported in the bottom panels as colored solid lines, vertically translated for the sake of the clarity. 

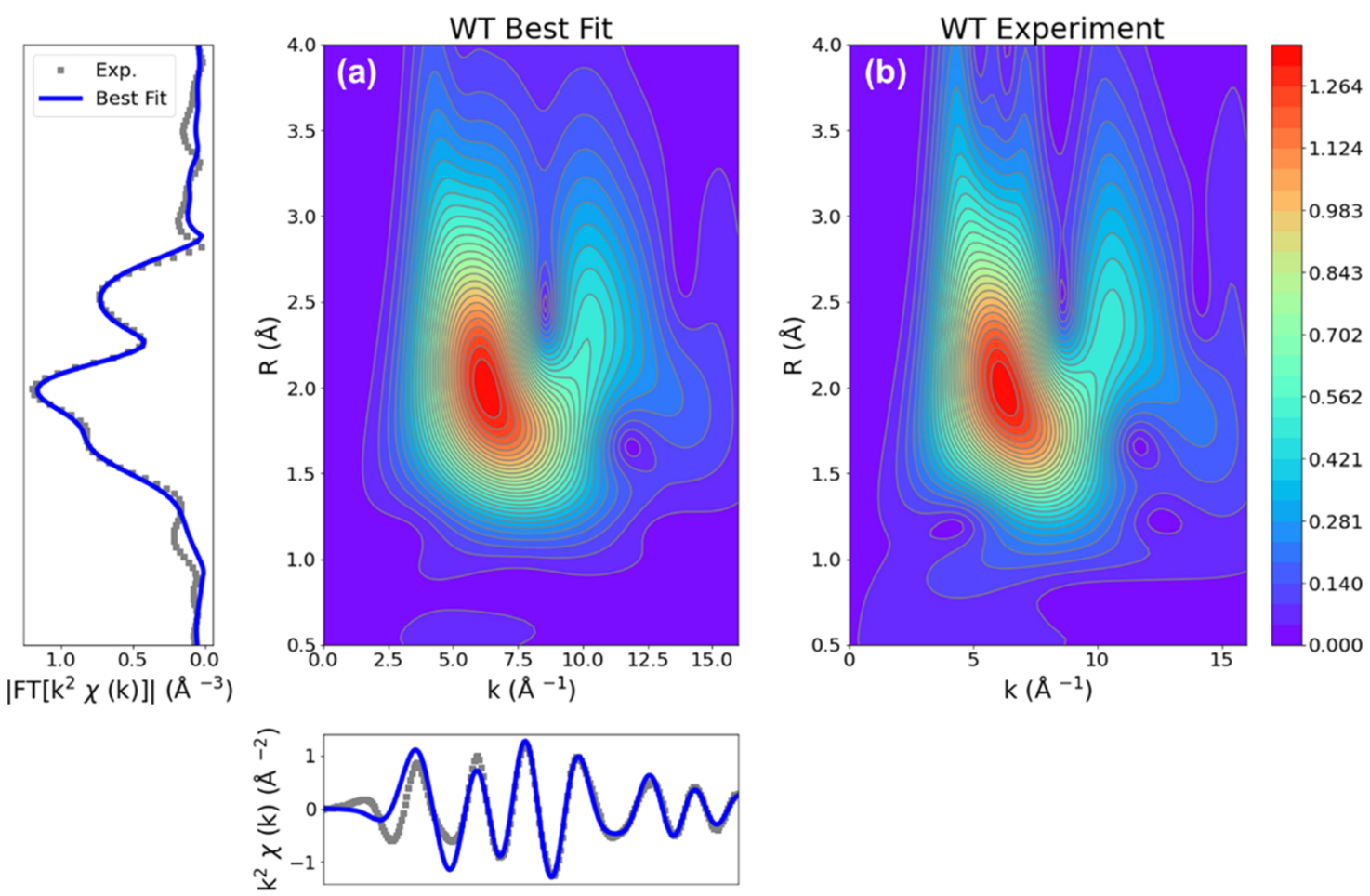

Figure 7. Plot of the EXAFS best-fit WT representation (phase uncorrected). The bottom and left panels report the comparison between the bestfit EXAFS curve, evaluated using the parameters found to minimize eq 3 , and the experimental spectrum plotted in the $k$ and $R$ (phaseuncorrected) spaces. The fit has been realized in the $\Delta k \times \Delta R=(0-16) \AA^{-1} \times(1-3) \AA 2 \mathrm{D}$ grid assuming a Hanning apodization window defined within 4 and $16 \AA^{-1}$. (b) WT representation of the experimental EXAFS signal. For both the WT representations, the same mother wavelet resolution parameters have been employed: $s=1$ and $\eta=5$.

Table 3. Best-Fit Parameters $\left(\mathrm{L}_{2}\right.$ Minimization Output) Obtained from the WT Representation, Minimizing the Target Function of eq S4 Considering the Set of Deformations Reported in Figure $S 9(\mathrm{a}-\mathrm{c})^{a}$

\begin{tabular}{lll}
\multicolumn{2}{c}{ best-fit parameters } & confidence intervals \\
$S_{0}^{2}$ & 1.21 & $0.94 \div 1.3$ \\
$\Delta E(\mathrm{eV})$ & -1.93 & $-5.60 \div 1.48$ \\
$\sigma_{\mathrm{C}}^{2}\left(\AA^{2}\right)$ & 0.004 & $0.0018 \div 0.008$ \\
$\sigma_{\mathrm{O}}^{2}\left(\AA^{2}\right)$ & 0.0055 & $0.0029 \div 0.01$ \\
$\sigma_{\mathrm{Cl}}^{2}\left(\AA^{2}\right)$ & 0.0048 & $0.0028 \div 0.0075$ \\
$\mathrm{Ru}-\mathrm{C}(\AA)$ & 1.9 & $1.88 \div 1.93$ \\
$\mathrm{Ru}-\mathrm{O}(\AA)$ & 3.036 & $3.01 \div 3.07$ \\
$\mathrm{Ru}-\mathrm{Cl}(\AA)$ & 2.405 & $2.38 \div 2.43$
\end{tabular}

${ }^{a}$ On the right columns are indicated the parameter uncertainties obtained through the F-test approach fixing a confidence level of $68 \%$ for 7 D.O.F.

$0.004 \pm 0.006 \AA$, see Figure $8 \mathrm{~b}$. However, in this case, the $\% R$ factor is increased to $0.9 \%$, similarly to what it has been found through the fitting procedure described in the precedent section. The reason for the discrepancy between the two approaches must be found in the molecular geometry dependence of the amplitudes and of phases of the SS and MS paths generated by the code, which are calculated, through an $a b$ initio approach, just one time for the structure with a $\mathrm{Ru}-\mathrm{C}$ bond length of $1.70 \AA$. These curves remain in fact unaltered for the extension of the $\mathrm{Ru}-\mathrm{C}$ bond to $\Delta d_{\mathrm{C}}=+0.20$ $\AA$, as already critically noticed by Ravel. ${ }^{29}$ This approximation is instead not applied in our method, where each selected path is numerically approximated for the continuous variations of the geometric parameters, providing a more accurate refinement of the structure.

If the starting $\mathrm{Ru}-\mathrm{C}$ is increased to $2.2 \AA$ keeping the $\mathrm{Ru}-\mathrm{O}$ distance unchanged, the related $R$-factor increases to $13 \%$ (see Figure $8 \mathrm{c}$ ). The fitted $\mathrm{R}_{\mathrm{Ru}-\mathrm{C}}$ is optimized to $2.36 \pm 0.07 \AA$, which is clearly unphysically large, indicating how the Artemis code, using the amplitudes and phases generated starting from the structure with a $\mathrm{Ru}-\mathrm{C}=2.2 \AA$, is not able to fit properly the experimental spectrum. Herein, the geometry dependence of the paths can be further underlined involving in a fourth refinement this structure $(\mathrm{Ru}-\mathrm{C}$ of $2.2 \AA)$ but taking the independent $\mathrm{Ru}-\mathrm{C}$ path from the second structure $\left(R_{\mathrm{Ru}-\mathrm{C}}=1.90 \AA\right)$ and assigning to it an independent $\Delta E_{0}$. This fit, reported in Figure 8d, employs one parameter more than the ones reported before, and it appears characterized by an $R$-factor of $1.2 \%$ for a $\mathrm{Ru}-\mathrm{C}$ distance of $1.88 \pm 0.02 \AA$. This quantity is higher than the one realized with the model initialized with a $\mathrm{Ru}-\mathrm{C}=1.9 \AA$, and the difference is attributable to the MS contributions.

Concluding, these examples demonstrate how the choice of the starting geometry affects not only the values of the fitted parameters but also the overall fitting quality, showing, unambiguously, that finding the best fit is not a trivial task, especially when MS paths are involved. In this representative example, the developed ML approach appears to be highly beneficial.

\section{CONCLUSIONS}

We have developed a novel fitting approach based on ML that aims to improve the classic MS EXAFS fit methodology. This approach allows one to overcome the known issue, proper of the standard EXAFS fitting procedure, related to the 

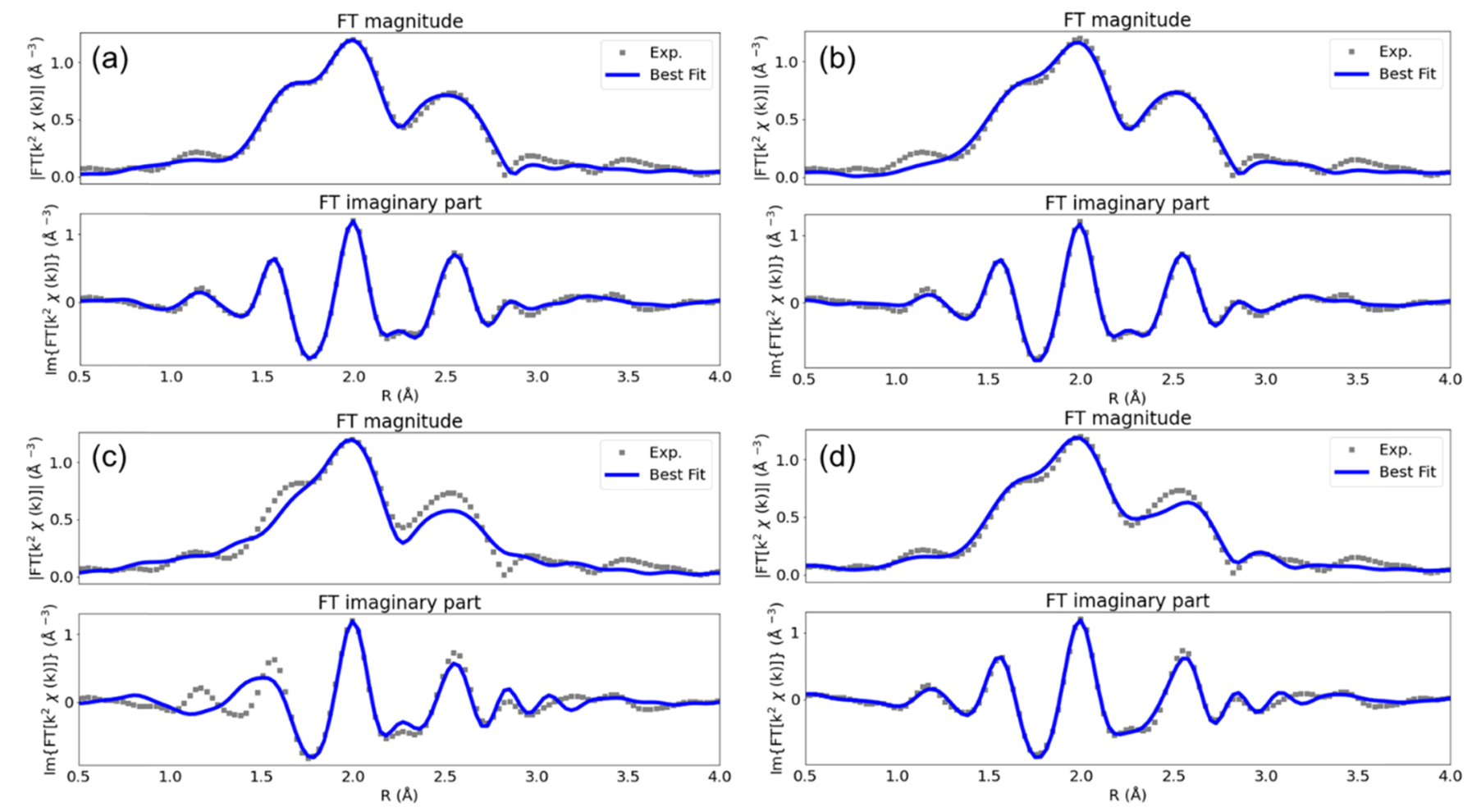

Figure 8. Fit of the experimental EXAFS signal obtained using the $\mathrm{Ru}(\mathrm{CO})_{3} \mathrm{Cl}_{3}$ model starting from different values of the $\mathrm{Ru}-\mathrm{C}$ distance and with a common $\mathrm{Ru}-\mathrm{O}$ bond length set to $3.0 \AA$. (a) $\mathrm{Ru}-\mathrm{C}$ distance of $1.70 \AA$, corresponding to the minimal contraction of the $\mathrm{Ru}-\mathrm{C}$ bond $\left(\Delta d_{\mathrm{C}}=-0.2 \AA\right)$. (b) $\mathrm{Ru}-\mathrm{C}$ distance of $1.90 \AA$. (c) $\mathrm{Ru}-\mathrm{C}$ distance set to $2.2 \AA\left(\Delta d_{\mathrm{C}}=+0.3 \AA\right)$. (d) Fit of the EXAFS signal based on the model with the $\mathrm{Ru}-\mathrm{C}$ bond distance set to $2.2 \AA$ but employing the $\mathrm{Ru}-\mathrm{C}$ path generated for the $\mathrm{Ru}-\mathrm{C}$ distance of $1.90 \AA$.

dependency of the theoretical phases, amplitudes, and electron mean free paths on the input geometry, which has been shown to be a critical aspect for the MS process. Unlike other fitting approaches, the proposed methodology allows optimizing the parameters directly describing the $3 \mathrm{D}$ geometry without the requirement of any complex parametrization, leading to a strong simplification in the EXAFS analysis. We have demonstrated the validity and advantages of this novel approach fitting the EXAFS data for two representative Auand $\mathrm{Ru}$-based molecular complexes.

\section{ASSOCIATED CONTENT}

OSupporting Information

Part S1. Fitting routine: Multidimensional coarse grid plot (Figure S1); equivalence criterion scheme (Figure S2); plot of the IHS point distributions (Figure S3); and $\mathrm{KAu}(\mathrm{CN})_{2}$ path datasets (Figure S4), Part S2: Experimental data, Part S3: EXAFS analysis of the $\mathrm{KAu}(\mathrm{CN})_{2}$ spectrum: Set of deformations employed in the fit of the $\mathrm{KAu}(\mathrm{CN})_{2}$ complex (Section S3.1, Figure S5); quality of prediction associated with each ML path function $\left(\mathrm{KAu}(\mathrm{CN})_{2}\right.$ complex case) (Section 3.2, Table $\mathrm{S} 1$ ); curve-wave amplitude analysis of the main MS processes for the $\mathrm{KAu}(\mathrm{CN})_{2}$ case (Section S3.3, Figure S6 and Figure S7); and error curves evaluated for the variation of the $\alpha$ and $\beta$ parameters around the minimum found by the refinement (Section S3.4 and Figure S8), Part S4. EXAFS analysis of $\left[\mathrm{RuCl}_{2}(\mathrm{CO})_{3}\right]_{2}$ complex: Set of deformations employed in the fit of the $\left[\mathrm{RuCl}_{2}(\mathrm{CO})_{3}\right]_{2}$ complex (Figure S9) and quality of prediction associated with each ML path function (the $\left[\mathrm{RuCl}_{2}(\mathrm{CO})_{3}\right]_{2}$ complex case) (Table S2), Part S5: EXAFS WT fitting routine description: EXAFS WT fit of the $\mathrm{KAu}(\mathrm{CN})_{2}$ complex (Section 5.1, Table S3 and Figure S10), and Part 6: Validation of the ML-refined structures through the classic EXAFS fitting procedure: $\mathrm{KAu}(\mathrm{CN})_{2}$ case (Section S6.1, Figure S11 and Table S4) and $\left[\mathrm{RuCl}_{2}(\mathrm{CO})_{3}\right]_{2}$ case (Section 6.2, Figure S12, and Table S5) (PDF)

\section{AUTHOR INFORMATION}

\section{Corresponding Authors}

A. Martini - The Smart Materials Research Institute, Southern Federal University, 344090 Rostov-on-Don, Russia; Department of Chemistry, University of Torino, 10125 Torino, Italy; 이이이.org/0000-0001-8820-2157; Email: andrea.martini@unito.it

A. L. Bugaev - The Smart Materials Research Institute, Southern Federal University, 344090 Rostov-on-Don, Russia; Southern Scientific Centre, Russian Academy of Sciences, 344006 Rostov-on-Don, Russia; Email: abugaev@sfedu.ru

\section{Authors}

S. A. Guda - The Smart Materials Research Institute, Southern Federal University, 344090 Rostov-on-Don, Russia; Institute of mathematics, mechanics and computer science, Southern Federal University, 344090 Rostov-on-Don, Russia; (1) orcid.org/0000-0002-2398-1847

A. A. Guda - The Smart Materials Research Institute, Southern Federal University, 344090 Rostov-on-Don, Russia; (1) orcid.org/0000-0002-6941-4987 
E. Priola - Department of Chemistry, University of Torino, 10125 Torino, Italy; CrisDi, Interdepartemental Center for Crystallography, University of Turin, Torino, Via P. Giuria 7 I-10125, Italy; 이이이.org/0000-0002-0270-738X

E. Borfecchia - Department of Chemistry, University of Torino, 10125 Torino, Italy; (1) orcid.org/0000-0001-83748329

S. Smolders - Department of Microbial and Molecular Systems (M2S); Centre for Membrane separations, Adsorption, Catalysis and Spectroscopy for Sustainable Solutions (cMACS), KU Leuven, 3001 Leuven, Belgium

K. Janssens - Department of Microbial and Molecular Systems (M2S); Centre for Membrane separations, Adsorption, Catalysis and Spectroscopy for Sustainable Solutions (cMACS), KU Leuven, 3001 Leuven, Belgium; 이이.org/ 0000-0002-7194-2623

D. De Vos - Department of Microbial and Molecular Systems (M2S); Centre for Membrane separations, Adsorption, Catalysis and Spectroscopy for Sustainable Solutions (cMACS), KU Leuven, 3001 Leuven, Belgium; (1) orcid.org/ 0000-0003-0490-9652

A. V. Soldatov - The Smart Materials Research Institute, Southern Federal University, 344090 Rostov-on-Don, Russia

\section{Notes}

The authors declare no competing financial interest.

\section{ACKNOWLEDGMENTS}

A.M. acknowledges project n. 2017KKP5Z PRIN-2017 MOSCATo (Cutting-edge X-ray methods and models for the understanding of surface site reactivity in heterogeneous catalysts and sensors). A.L.B. acknowledges the Russian Foundation for Basic Research Grant \#19-32-60083 for funding the research in the field of $\mathrm{ML}$ analysis of X-ray absorption data. A.L.B., S.A.G., A.A.G., and A.V.S. acknowledge the Russian Science Foundation (joint RSF-FWO grant \# 20-43-01015) for funding the investigation of Ru-based systems. S.S., K.J., and D.D.V. acknowledge the Flemish government (FWO, project G0F2320N) for funding. The authors are grateful to $\mathrm{K}$. A. Lomachenko for his help during the $\mathrm{Au} \mathrm{L}_{3}$-edge XAS experiments at the BM23 beamline of the ESRF synchrotron and to C. La Fontaine and V. Briois for their support during the $\mathrm{Ru}$ K-edge measurements at the ROCK beamline of SOLEIL, which is benefiting from a public grant overseen by the French National Research Agency as part of the "Investissements d'Avenir" program (Reference: ANR-10-EQPX-45).

\section{ABBREVIATIONS}

EXAFS, extended X-ray absorption fine structure; SS, single scattering; MS, multiple scattering; DW, Debye-Waller; ML, machine learning; FT, Fourier transform; WT, wavelet transform; D.O.F, degree of freedom; CV, cross validation.

\section{REFERENCES}

(1) Rehr, J. J.; Albers, R. C. Rev. Mod. Phys. 2000, 72, 621.

(2) Van Bokhoven, J. A.; Lamberti, C. X-ray absorption and X-ray emission spectroscopy: theory and applications. John Wiley \& Sons, 2016; Vol. 1.

(3) Sayers, D. E.; Stern, E. A.; Lytle, F. W. Phys. Rev. Lett. 1971, 27, 1204.
(4) Seah, M. P.; Dench, W. A. Surf. Interface Anal. 1979, 1, 2.

(5) Frenkel, A. I.; Stern, E. A.; Qian, M.; Newville, M. Phys. Rev. B 1993, 48, 12449.

(6) Ravel, B.; Newville, M. J. Synchrotron. Radiat. 2005, $12,537$.

(7) Zabinsky, S. I.; Rehr, J. J.; Ankudinov, A.; Albers, R. C.; Eller, M. J. Phys. Rev. B Condens. Matter 1995, 52, 2995.

(8) Lee, P. A.; Pendry, J. B. Phys. Rev. B 1975, 11, 2795.

(9) Frenkel, A.; Stern, E. A.; Voronel, A.; Qian, M.; Newville, M. Phys. Rev. Lett. 1993, 71, 3485.

(10) Frenkel, A.; Stern, E. A.; Voronel, A.; Qian, M.; Newville, M. Phys. Rev. B 1994, 49, 11662.

(11) Frenkel, A.; Voronel, A.; Katzir, A.; Newville, M.; Stern, E. A. Phys. B Condens. Matter 1995, 208-209, 334-336.

(12) Frenkel, A. I.; Stern, E. A.; Voronel, A.; Heald, S. M. Solid State Commun. 1996, 99, 67.

(13) Filipponi, A.; di Cicco, A. Phys. Rev. B Condens. Matter 1995, $52,15135$.

(14) Filipponi, A.; di Cicco, A.; Natoli, C. R. Phys. Rev. B Condens. Matter 1995, 52, 15122.

(15) D’Angelo, P.; di Nola, A.; Filipponi, A.; Pavel, N. V.; Roccatano, D. J. Chem. Phys. 1994, 100, 985.

(16) D’Angelo, P.; Barone, V.; Chillemi, G.; Sanna, N.; MeyerKlaucke, W.; Pavel, N. V. J. Am. Chem. Soc. 2002, 124, 1958.

(17) Kuzmin, A.; Evarestov, R. A. J. Phys. Condens. Matter 2009, 21, No. 055401.

(18) Di Cicco, A.; Trapananti, A.; Faggioni, S.; Filipponi, A. Phys. Rev. Lett. 2003, 91, No. 135505.

(19) Timoshenko, J.; Kuzmin, A.; Purans, J. Comput. Phys. Commun. 2012, 183, 1237.

(20) Calvin, S. XAFS for Everyone. CRC Press, 2013, DOI: 10.1201/ b14843.

(21) Vlaic, G.; Olivi, L. Croat. Chem. Acta 2004, 77, 427.

(22) Hudson, E. A.; Allen, P. G.; Terminello, L. J.; Denecke, M. A.; Reich, T. Phys. Rev. B 1996, 54, 156.

(23) Timoshenko, J.; Anspoks, A.; Cintins, A.; Kuzmin, A.; Purans, J.; Frenkel, A. I. Phys. Rev. Lett. 2018, 120, No. 225502.

(24) Timoshenko, J.; Wrasman, C. J.; Luneau, M.; Shirman, T.; Cargnello, M.; Bare, S. R.; Aizenberg, J.; Friend, C. M.; Frenkel, A. I. Nano Lett. 2019, 19, 520.

(25) Guda, A. A.; Guda, S. A.; Lomachenko, K. A.; Soldatov, M. A.; Pankin, I. A.; Soldatov, A. V.; Braglia, L.; Bugaev, A. L.; Martini, A.; Signorile, M.; Groppo, E.; Piovano, A.; Borfecchia, E.; Lamberti, C. Catal. Today 2019, 336, 3.

(26) Smolentsev, G.; Soldatov, A. J. Synchrotron. Radiat. 2006, 13, 19.

(27) Martini, A.; Guda, S. A.; Guda, A. A.; Smolentsev, G.; Algasov, A.; Usoltsev, O.; Soldatov, M. A.; Bugaev, A.; Rusalev, Y.; Lamberti, C.; Soldatov, A. V. Comput. Phys. Commun. 2020, 250, No. 107064.

(28) Guda, A. A.; Guda, S. A.; Martini, A.; Bugaev, A. L.; Soldatov, M. A.; Soldatov, A. V.; Lamberti, C. Radiat. Phys. Chem. 2020, 175, No. 108430.

(29) Ravel, B. In 14th International Conference on X-Ray Absorption Fine Structure; DiCicco, A., Filipponi, A., Eds.; Iop Publishing Ltd: Bristol, 2009, p. 190.

(30) Ravel, B. In X-Ray Absorption and X-Ray Emission Spectroscopy; Van Bokhoven, J. A., Lamberti, C., Eds.; Wiley Online Books: 2016, p. 281.

(31) Beachkofski, B.; Grandhi, R. In 43rd AIAA/ASME/ASCE/ AHS/ASC Structures, Structural Dynamics, and Materials Conference, 2002.

(32) Moré, J. J. In Numerical Analysis; Watson, G. A., Ed.; Springer Berlin Heidelberg: Berlin, Heidelberg, 1978, p. 105.

(33) Nelder, J. A.; Mead, R. Comput. J. 1965, 7, 308.

(34) James, F.; Roos, M. Comput. Phys. Commun. 1975, 10, 343.

(35) Powell, M. J. D. Comput. J. 1964, 7, 155.

(36) Press, W. H.; Teukolsky, S. A.; Vetterling, W. T.; Flannery, B. P. Numerical recipes in $C$ (2nd ed.): the art of scientific computing. Cambridge University Press, 1992. 
(37) Bunker, G. Introduction to XAFS: A Practical Guide to X-ray Absorption Fine Structure Spectroscopy. Cambridge University Press: Cambridge, 2010, DOI: 10.1017/CBO9780511809194.

(38) Kuzmin, A. Phys. B 1995, 208-209, 175.

(39) Newville, M.; Stensitzki, T.; Allen, D. B.; Ingargiola, A. Zenodo 2014.

(40) Joyner, R. W.; Martin, K. J.; Meehan, P. J. Phys. C Sol. State Phys. 1987, 20, 4005.

(41) Greaves, G. N.; Dent, A. J.; Dobson, B. R.; Kalbitzer, S.; Pizzini, S.; Muller, G. Phys. Rev. B 1992, 45, 6517.

(42) Bevington, P. R.; Robinson, D. K. Data reduction and error analysis. McGraw-Hill: New York, 2003.

(43) Taylor, J. R. Error analysis. Univ Science Books: Sausalito, California, 1997.

(44) Timoshenko, J.; Kuzmin, A. Comput. Phys. Commun. 2009, 180, 920.

(45) El Osta, R.; Frigoli, M.; Marrot, J.; Medina, M. E.; Walton, R. I.; Millange, F. Cryst. Growth Des. 2012, 12, 1531.

(46) Millange, F.; Medina, M. I.; Guillou, N.; Férey, G.; Golden, K. M.; Walton, R. I. Angew. Chem. Int. Ed. 2010, 49, 763.

(47) Nangia, A.; Desiraju, G. R. Acta Crystallogr. A 1998, 54, 934.

(48) Priola, E.; Volpi, G.; Rabezzana, R.; Borfecchia, E.; Garino, C.; Benzi, P.; Martini, A.; Operti, L.; Diana, E. Inorg. Chem. 2020, 59, 203.

(49) Stavitski, E.; Goesten, M.; Juan-Alcañiz, J.; Martinez-Joaristi, A.; Serra-Crespo, P.; Petukhov, A. V.; Gascon, J.; Kapteijn, F. Angew. Chem. Int. Ed. 2011, 50, 9624.

(50) van Vleet, M. J.; Weng, T.; Li, X.; Schmidt, J. R. Chem. Rev. 2018, 118, 3681.

(51) Alexandrov, E. V.; Virovets, A. V.; Blatov, V. A.; Peresypkina, E. V. Chem. Rev. 2015, 115, 12286.

(52) Cairns, A. B.; Catafesta, J.; Levelut, C.; Rouquette, J.; van der Lee, A.; Peters, L.; Thompson, A. L.; Dmitriev, V.; Haines, J.; Goodwin, A. L. Nat. Mater. 2013, 12, 212.

(53) Cui, G.; Cao, X.-Y.; Fang, W.-H.; Dolg, M.; Thiel, W. Angew. Chem. Int. Ed. 2013, 52, 10281.

(54) Fernandez, E. J.; Laguna, A.; Lopez-De-Luzuriaga, J. M. Dalton Trans. 2007, 1969.

(55) Lefebvre, J.; Batchelor, R. J.; Leznoff, D. B. J. Am. Chem. Soc. 2004, 126, 16117.

(56) Sayers, D. E.. Error Reporting Recommendations: A Report of the Standards and Criteria Committee. International X-ray Absorption Society, 2000, p. 1.

(57) Kido, D.; Uemura, Y.; Wakisaka, Y.; Ariga-Miwa, H.; Takakuasgi, S.; Asakura, K. J. Surf. Sci. Nanotechnol. 2020, 18, 249.

(58) Stalpaert, M.; Janssens, K.; Marquez, C.; Henrion, M.; Bugaev, A. L.; Soldatov, A. V.; De Vos, D. ACS Catal. 2020, 10, 9401.

(59) Moreno, M. A.; Haukka, M.; Kallinen, M.; Pakkanen, T. A. Appl. Organomet. Chem. 2006, 20, 51.

(60) Sun, D.; Gao, Y.; Fu, J.; Zeng, X.; Chen, Z.; Li, Z. Chem. Commun. 2015, 51, 2645. 